\title{
Construction of Mathematical Model for the Bending State of Braided Wire Rope
}

\author{
Zhao Ping $^{1, \text { a }}$, Wang Hui ${ }^{2, b^{*}}$,Tian Xiangsheng ${ }^{3, c}$ and ZHAO Xia \\ ${ }^{1}$ School of Mechanical Engineering, University of Jinan, Jinan 250022, China \\ ${ }^{2}$ School of Mechanical Engineering, University of Jinan, Jinan 250022, China \\ ${ }^{3}$ School of Mechanical Engineering, University of Jinan, Jinan 250022, China \\ ${ }^{4}$ School of Mechanical Engineering, University of Jinan, Jinan 250022, China \\ aemail: \\ 18753136032@163.com, bemail:me_wangh@ujn.edu.cn, cemail:maxwell_tian@163.com, demail:jz \\ 1015zhaoxia@163.com
}

Keywords: Braided Wire Rope; Bending; Mathematical Model

\begin{abstract}
The characteristics of spatial structure and winding forming principle of braided wire rope under bending condition are analyzed. Establish the local coordinate system of the wire rope strand and the center line of the strand.The transformation matrix of each coordinate system is derived by using the theory of differential geometry and spatial coordinate transformation,and the mathematical model of the bending state of wire rope strand and wire center line is established. The geometry model of the model which lay a theoretical foundation for the analysis of the mechanical properties of the braided wire rope is established on the basis of mathematical model.
\end{abstract}

\section{Introduction}

The wire rope can be divided into twisted round strand wire rope and braided wire rope twoaccording to its forming in different ways as shown in figure 1 , 2.Twisting wire rope is made from steel wire strands and the steel wire strands are made by wire-steel. The braided wire rope is composed of a set of left to twist and a set of right to twist single strand round strand wire rope through a certain rule, The torque, which has the same direction and the opposite direction under the action of tension, is widely used in the power grid construction project as the guide line and the traction rope.Because the steel wire rope is bending when around the traction machine of its traction in the process of erecting cable, Its stress is more complex than the straight statet , the fatigue, wear, broken wires, etc. often cause accidents, has been the key problems of power transmission enterprise stringing construction safety [1]. Therefore, it is necessary to study the stress and strain condition of the braided wire rope.

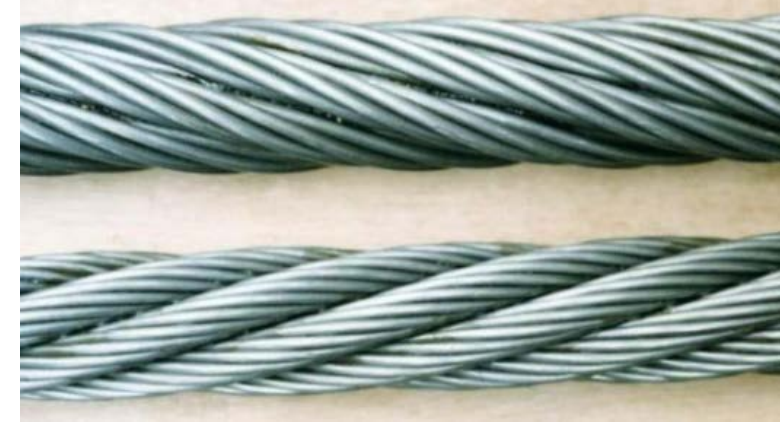

Fig.1. Twisted wire rope

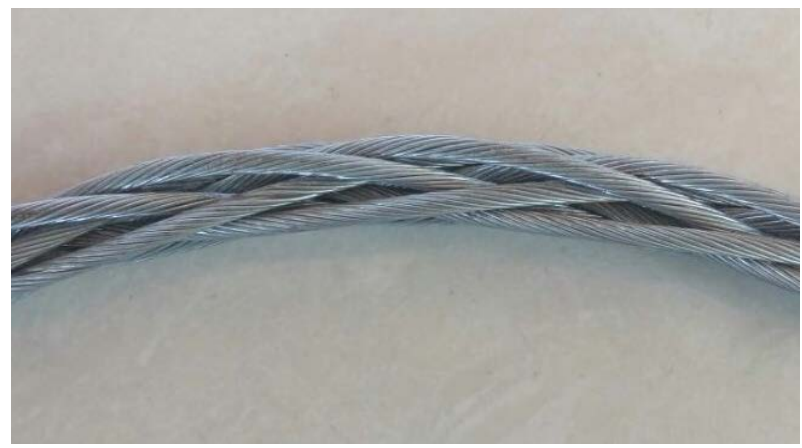

Fig.2. Braided wire rope

Foreign scholars Hobbs R E, Nabijou S [2][3] established mathematica model for twisting wire rope in the upright and bending condition by applying the theory of differential geometry and space coordinate transformation relation, and studied the curvature change of the steel wire rope .Wu Juan, taking the right direction of the wire rope as an example, the mathematical model of the wire rope in the bending state was established, and the distribution of the equivalent stress and deformation of 
different twist to the wire rope was analyzed [4]. Guo Wei established the mathematical model of the wire rope in the bending state by using the theory of spatial coordinate transformation [5].

To sum up, the domestic and foreign scholars have conducted a lot of research for twisting wire rope, and achieved certain results, but for the braided wire rope has not been found.relevant studies. In view of this, this paper establishes the mathematical model of the braided wire rope in the bending state with the space coordinate transformation theory, and lays the foundation for the stress analysis of the wire rope.

\section{Establishment of Mathematical Model for Bending State of Braided Wire Rope}

\section{Construction of Mathematical Model of the Steel Wire Rope Strand Center.}

Braided wire rope around the drum of the tractor In the process of installation and construction , the central axis of the steel wire rope is bent into a curved stateDue to the winding action of the winding drum, The center line of each strand in the wire rope is a space spiral line which is curved around the circular arc and the side wire of each side of the wire rope is two spiral lines of bending in space.

Figure 3 is the winding forming principle of the space spiral winding of the braided wire rope which is wound around the circular arc.

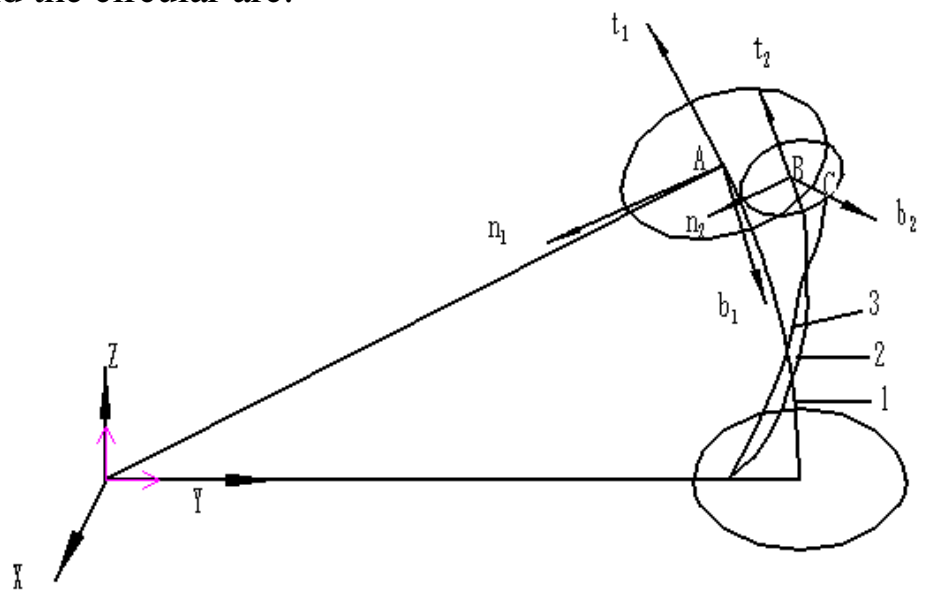

1 Center line of wire rope; 2 Central line of wire rope strand; 3 Central line of steel wire in strand

Fig.3. Winding forming principle of braided wire rope with circular arc bending

In Figure $3: n_{1}$ is the unit vector of the point $A$ along the normal direction, $t_{1}$ is the unit vector of the point $\mathrm{A}$ along the tangent direction, $b_{1}$ is the vector of point $\mathrm{A}$ of the arc, $n_{2}$ is the unit vector of the point $\mathrm{B}$ along the normal direction in the space of the curved circular arc curved space, $t_{2}$ is the unit vector of the point $\mathrm{B}$ along the tangent direction in the space of curved curved space, $b_{2}$ is the method vector from the point $\mathrm{B}$ in the space of the curved space of the circular arc.

Figure 3 is the winding forming principle of the space spiral winding of the braided wire rope which is wound around the circular arc. The overall coordinate system is $X Y Z$, the local coordinate system of the space spiral line is $n_{1}-b_{1}-t_{1}$, and the local coordinate system of the curved spiral curve of the spiral line is $n_{2}-b_{2}-t_{2}$.

The space curve equation of circular arc is:

$$
R_{\text {circle }}=\left\{\begin{array}{l}
x_{1} \\
y_{1} \\
z_{1}
\end{array}\right\}=\left\{\begin{array}{l}
0 \\
r_{L} \cos \theta_{L} \\
r_{L} \sin \theta_{L}
\end{array}\right\}
$$

According to the spatial coordinate transformation, Stock center line of the point $B$ where the local coordinate system $n_{1}-b_{1}-t_{1}$ conversion to the overall coordinate system $X Y Z$ conversion matrix $T_{1}$ : 


$$
T_{1}=\left[n_{1}, b_{1}, t_{1}\right]=\left[\begin{array}{ccc}
0 & 1 & 0 \\
-\cos \theta_{L} & 0 & -\sin \theta_{L} \\
-\sin \theta_{L} & 0 & \cos \theta_{L}
\end{array}\right]
$$

Rope strand right to weave, conversion matrix of the local coordinate system $n_{2}-b_{2}-t_{2}$ converted to the local coordinate system $n_{1}-b_{1}-t_{1}$ is $T_{2 \text { right }}^{\prime}$, conversion matrix of local coordinate system $n_{2^{-}}$ $b_{2}-t_{2}$ conversion to the overall coordinate system $X Y Z$ is $T_{2 \text { right }}, T_{2 \text { right }}=T_{1} T_{2 \text { right }}^{\prime}$.

Vector expression of point $B$ on the curved primary spatial spiral line in the right direction in the local coordinate system $n_{1}-b_{1}-t_{1}$ :

$$
\overrightarrow{A B^{\prime}}=\left\{\begin{array}{l}
a \cos \left(\theta+\theta_{0}\right) \\
b \sin \left(\theta+\theta_{0}\right) \\
0
\end{array}\right\}
$$

In the above formula: $a$ is the length of the major axis of the ellipse is $1 / 2, b$ is $1 / 2$ the length of the short axis of the ellipse, $c$ related parameters and wire rope pitch, namely c=P/2 PI, $\theta$ is elliptic theta centrifugal angle, $\theta_{0}$ is initial braid angle of rope strand.

Vector expression of point $B$ on the curved primary spatial spiral line in the right direction in the global coordinate system $X Y Z$ is $\overrightarrow{A B}$ :

$$
\overrightarrow{A B}=T_{1} \overrightarrow{A B^{\prime}}=\left[\begin{array}{l}
b \sin \left(\theta+\theta_{0}\right) \\
-a \cos \theta_{\mathrm{L}} \cos \left(\theta+\theta_{0}\right) \\
-a \sin \theta_{\mathrm{L}} \cos \left(\theta+\theta_{0}\right)
\end{array}\right]
$$

The vector equation of a single spiral line in the space around a circular arc. is $P_{1 \text { right }}$ :

$$
P_{\text {1right }}=R_{\text {circle }}+\overrightarrow{A B^{\prime}}=\left[\begin{array}{l}
b \sin \left(\theta+\theta_{0}\right) \\
r_{\mathrm{L}} \cos \theta_{\mathrm{L}}-a \cos \theta_{\mathrm{L}} \cos \left(\theta+\theta_{0}\right) \\
r_{\mathrm{L}} \sin \theta_{\mathrm{L}}-a \sin \theta_{\mathrm{L}} \cos \left(\theta+\theta_{0}\right)
\end{array}\right]
$$

In the same way, the rope strands are left to weave, The vector equation of a single spiral line in the space around a circular is $P_{1 l e f t}$ :

$$
P_{1 \text { left }}=R_{\text {circle }}+\overrightarrow{A B}=\left[\begin{array}{l}
a \sin \left(-\theta+\theta_{0}\right) \\
r_{\mathrm{L}} \cos \theta_{\mathrm{L}}-\mathrm{b} \cos \theta_{\mathrm{L}} \cos \left(-\theta+\theta_{0}\right) \\
r_{\mathrm{L}} \sin \theta_{L}-\mathrm{b} \sin \theta_{\mathrm{L}} \cos \left(-\theta+\theta_{0}\right)
\end{array}\right]
$$

\section{Construction of Mathematical Model of Central Line of Steel Wire in Strand.}

The wire winding by left side in the strand, Vector expression of point $\mathrm{C}$ on two times spiral line in the curved space in the local coordinate system of $n_{2}-b_{2}-t_{2}$ is $\overrightarrow{B C^{\prime}}$ :

$$
\overrightarrow{B C^{\prime}}=\left\{\begin{array}{l}
r_{s} \cos \left(-\theta_{s}+\theta_{s 0}\right) \\
r_{s} \sin \left(-\theta_{s}+\theta_{s 0}\right) \\
0
\end{array}\right\}
$$

In the above formula: $r_{\mathrm{s}}$ is the line wire in stocks in the spiral winding radius, $\theta_{s}$ is the spiral steel wire side winding angle, $\theta_{\mathrm{s} 0}$ is the initial line of wire winding angle.

The transformation matrix of the local coordinate system $n_{2}-b_{2}-t_{2}$ to the global coordinate system $X Y Z$ is: $\overrightarrow{B C}=T_{2 \text { right }} \overrightarrow{B C^{\prime}}$.

The vector equations of the two spiral lines in the curved space is $P_{2 \text { right } x \text { left }}$ : 


$$
\begin{aligned}
& {\left[\quad b \sin \left(\theta+\theta_{0}\right)-\frac{r_{s} b \sin \left(\theta+\theta_{0}\right) \cos \left(-\theta_{s}+\theta_{s o}\right)}{\sqrt{a^{2} \cos ^{2}\left(\theta+\theta_{0}\right)+b^{2} \sin ^{2}\left(\theta+\theta_{0}\right)}}-\right.} \\
& P_{\text {2right } \times \text { left }}=R_{\text {circle }}+\overrightarrow{A B}+\overrightarrow{B C}=r_{L} \cos \theta_{L}-\operatorname{acos} \theta_{\mathrm{L}} \cos \left(\theta+\theta_{0}\right)+\frac{a r_{s} \cos \left(\theta+\theta_{0}\right) \cos \left(-\theta_{s}+\theta_{s o}\right) \cos \theta_{L}}{\sqrt{a^{2} \cos ^{2}\left(\theta+\theta_{0}\right)+b^{2} \sin ^{2}\left(\theta+\theta_{0}\right)}} \\
& r_{L} \sin \theta_{L}-\operatorname{asin} \theta_{\mathrm{L}} \cos \left(\theta+\theta_{0}\right) \frac{a r_{s} \sin \theta_{L} \cos \left(-\theta_{s}+\theta_{s o}\right) \cos \left(\theta+\theta_{0}\right)}{\sqrt{a^{2} \cos ^{2}\left(\theta+\theta_{0}\right)+b^{2} \sin ^{2}\left(\theta+\theta_{0}\right)}}+ \\
& \frac{a c r_{s} \cos \left(\theta+\theta_{0}\right) \sin \left(-\theta_{s}+\theta_{s o}\right)}{\sqrt{a^{2} \cos ^{2}\left(\theta+\theta_{0}\right)+b^{2} \sin ^{2}\left(\theta+\theta_{0}\right)} \sqrt{a^{2} \sin ^{2}\left(\theta+\theta_{0}\right)+b^{2} \cos ^{2}\left(\theta+\theta_{0}\right)+c^{2}}} \\
& \frac{b c r_{s} \sin \left(\theta+\theta_{0}\right) \sin \left(-\theta_{s}+\theta_{s o}\right) \cos \theta_{L}+r_{s} a b \sin \theta_{L} \sin \left(-\theta_{s}+\theta_{s o}\right)}{\sqrt{a^{2} \cos ^{2}\left(\theta+\theta_{0}\right)+b^{2} \sin ^{2}\left(\theta+\theta_{0}\right)} \sqrt{a^{2} \sin ^{2}\left(\theta+\theta_{0}\right)+b^{2} \cos ^{2}\left(\theta+\theta_{0}\right)+c^{2}}} \\
& \frac{-b c r_{s} \sin \left(\theta+\theta_{0}\right) \sin \left(-\theta_{s}+\theta_{s o}\right) \sin \theta_{L}+r_{s} a b \cos \theta_{L} \sin \left(-\theta_{s}+\theta_{s o}\right)}{\sqrt{a^{2} \cos ^{2}\left(\theta+\theta_{0}\right)+b^{2} \sin ^{2}\left(\theta+\theta_{0}\right)} \sqrt{a^{2} \sin ^{2}\left(\theta+\theta_{0}\right)+b^{2} \cos ^{2}\left(\theta+\theta_{0}\right)+c^{2}}}
\end{aligned}
$$

In the same way, the wire winding by left side in the strand, the vector equations of the two spiral lines in the curved space is $P_{\text {2left } x \text { right }}$ :

$$
\begin{aligned}
& {\left[a \sin \left(-\theta+\theta_{0}\right)-\frac{r_{s} a \cos \left(\theta+\theta_{0}\right) \cos \left(\theta_{s}+\theta_{s o}\right)}{\sqrt{a^{2} \cos ^{2}\left(\theta+\theta_{0}\right)+b^{2} \sin ^{2}\left(\theta+\theta_{0}\right)}}-\right.} \\
& P_{\text {2leftxright }}=R_{\text {circle }}+\overrightarrow{A B}+\overrightarrow{B C}=r_{L} \cos \theta_{L}-\mathrm{b} \cos \theta_{\mathrm{L}} \cos \left(-\theta+\theta_{0}\right)+\frac{b r_{s} \sin \left(\theta+\theta_{0}\right) \cos \left(\theta_{s}+\theta_{s o}\right) \cos \theta_{L}}{\sqrt{a^{2} \cos ^{2}\left(\theta+\theta_{0}\right)+b^{2} \sin ^{2}\left(\theta+\theta_{0}\right)}} \\
& r_{L} \sin \theta_{L}-\mathrm{b} \sin \theta_{\mathrm{L}} \cos \left(-\theta+\theta_{0}\right) \frac{b r_{s} \sin \theta_{L} \sin \left(\theta+\theta_{0}\right) \cos \left(-\theta_{s}+\theta_{s o}\right)}{\sqrt{a^{2} \cos ^{2}\left(\theta+\theta_{0}\right)+b^{2} \sin ^{2}\left(\theta+\theta_{0}\right)}}- \\
& \frac{b c r_{s} \cos \left(\theta+\theta_{0}\right) \sin \left(\theta_{s}+\theta_{s o}\right)}{\sqrt{a^{2} \cos ^{2}\left(\theta+\theta_{0}\right)+b^{2} \sin ^{2}\left(\theta+\theta_{0}\right)} \sqrt{a^{2} \sin ^{2}\left(\theta+\theta_{0}\right)+b^{2} \cos ^{2}\left(\theta+\theta_{0}\right)+c^{2}}} \\
& \frac{a r_{s} \cos \left(\theta+\theta_{0}\right) \sin \left(\theta_{s}+\theta_{s o}\right) \cos \theta_{L}-r_{s} a b \sin \theta_{L} \sin \left(\theta_{s}+\theta_{s o}\right)}{\sqrt{a^{2} \cos ^{2}\left(\theta+\theta_{0}\right)+b^{2} \sin ^{2}\left(\theta+\theta_{0}\right)} \sqrt{a^{2} \sin ^{2}\left(\theta+\theta_{0}\right)+b^{2} \cos ^{2}\left(\theta+\theta_{0}\right)+c^{2}}} \\
& \left.\frac{a r_{s} \cos \left(\theta+\theta_{0}\right) \sin \left(\theta_{s}+\theta_{s o}\right) \sin \theta_{L}+r_{s} a b \cos \theta_{L} \sin \left(\theta_{s}+\theta_{s o}\right)}{\sqrt{a^{2} \cos ^{2}\left(\theta+\theta_{0}\right)+b^{2} \sin ^{2}\left(\theta+\theta_{0}\right)} \sqrt{a^{2} \sin ^{2}\left(\theta+\theta_{0}\right)+b^{2} \cos ^{2}\left(\theta+\theta_{0}\right)+c^{2}}}\right]
\end{aligned}
$$

\section{Construction of Geometric Model for Bending State of Braided Wire Rope.}

On the basis of the mathematical model established above,a geometric model of the bending state of braided wire rope is established by SolidWorks as shown in Figure 4.

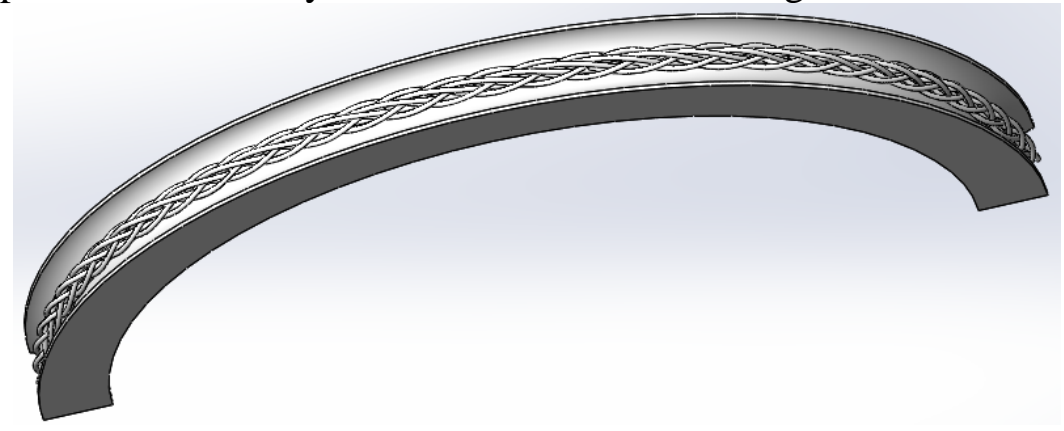

Fig.4. Geometry model of braided wire rope 


\section{Summary}

In this paper, the mathematical model of the wire rope in bending state is studied, and the space structure and winding forming principle of braided wire rope are analyzed, and the weaving process of braided wire rope are analyzed. The mathematical model of braided wire rope bending is established by using the theory of differential geometry and space coordinate transformation theory .This study will provide a theoretical basis for the analysis of the mechanical properties of braided wire rope in bending state.

\section{Funding}

The work was supported by the Science and Technology Development Project of Shandong Province [grant number 2014GGX103016].

\section{References}

[1] Shi Huanxue, Analysis of traction rope fault causes and preventive measures for tension stringing. [J] . Power supply technology for traction tension stringing. 2013,16(2) 137.

[2] Hobbs R E, Nabijou S, Changes in wire curvature as a wire rope is bent over a sheave. [J] . Journal of Strain Analysis for Engineering Design. 1995,30(2) 155-165.

[3] Nabijou S, Hobbs R E, Relative movements within wire ropes bent over sheaves. [J]. Journal of Strain Analysis for Engineering Design.1995, 30(4) 271-281.

[4] Wu Juan, Kou Ziming, Numerical simulation of stress and strain of steel wire in wire rope strand. [J] . Coal Journal. 2015,40(6) 1463-1468.

[5] Guo Wei, Lu Zhengxiong, Modeling theory and geometric realization of circular arc curved steel wire rope based on pro/e. [J] . China Mechanical Engineering. 2015,26(17) 2362-2368. 